\title{
Quaternionic Kähler and hyperKähler manifolds with torsion and twistor spaces
}

\author{
Stefan Ivanov * and Ivan Minchev \\ The Abdus Salam ICTP Preprint, December, 2001
}

\begin{abstract}
The target space of a $(4,0)$ supersymmetric two-dimensional sigma model with Wess-Zumino term has a connection with totally skew-symmetric torsion and holonomy contained in $\mathrm{Sp}(\mathrm{n}) \mathrm{Sp}(1)$ (resp. Sp(n)), QKT (resp. HKT)-spaces. We study the geometry of QKT, HKT manifold and their twistor spaces. We show that the Swann bundle of a QKT manifold admits a HKT structure with special symmetry if and only if the twistor space of the QKT manifold admits an almost hermitian structure with totally skew-symmetric Nijenhuis tensor, thus connecting two structures arising from quantum field theories and supersymmetric sigma models with WessZumino term. We discovered that a HKT manifold has always co-closed Lee form. Applying this property to compact HKT manifold we get information about the plurigenera.
\end{abstract}

Running title: Quaternionic Kähler with torsion

Keywords. Almost Quaternionic, Hyper Hermitian, Quaternionic Kähler, Torsion, Twistors.

AMS Subject Classification: Primary 53C25, Secondary 53C15, 53C56, 32L25, 57S25

\section{Introduction and statement of the results}

An almost hyper complex structure on a 4 n-dimensional manifold $M$ is a triple $H=\left(J_{\alpha}\right), \alpha=1,2,3$, of almost complex structures $J_{\alpha}: T M \rightarrow T M$ satisfying the quaternionic identities $J_{\alpha}^{2}=-i d$ and $J_{1} J_{2}=-J_{2} J_{1}=J_{3}$. When each $J_{\alpha}$ is a complex structure, $H$ is said to be a hyper complex structure on $M$.

An almost quaternionic structure on $M$ is a rank-3 subbundle $Q \subset \operatorname{End}(T M)$ which is locally spanned by almost hypercomplex structure $H=\left(J_{\alpha}\right)$; such a locally defined triple $H$ will be called an admissible basis of $Q$. A linear connection $\nabla$ on $T M$ is called quaternionic connection if $\nabla$ preserves $Q$, i.e. $\nabla_{X} \sigma \in \Gamma(Q)$ for all vector fields $X$ and smooth sections $\sigma \in \Gamma(Q)$. An almost quaternionic structure is said to be quaternionic if there is a torsion-free quaternionic connection.

* Partially supported by Contract MM 809/1998 with the Ministry of Science and Education of Bulgaria, Contract 353/2000 with the University of Sofia "St. Kl. Ohridski", The European Contract Human Potential Programme, Research Training Network HPRN-CT-2000-00101". 
A $Q$-hermitian metric is a Riemannian metric which is Hermitian with respect to each almost complex structure in $Q$. An almost quaternionic (resp. quaternionic) manifold with Q-hermitian metric is called an almost quaternionic Hermitian (resp. quaternionic hermitian) manifold

For $n=1$ an almost quaternionic structure is the same as an oriented conformal structure and it turns out to be always quaternionic. When $n \geq 2$, the existence of torsion-free quaternionic connection is a strong condition which is equivalent to the 1-integrability of the associated $\mathrm{GL}(\mathrm{n}, \mathrm{H}) \mathrm{Sp}(1)$ structure [11, 34, 43]. If the Levi-Civita connection of a quaternionic hermitian manifold $(M, g, Q)$ is a quaternionic connection then $(M, g, Q)$ is called Quaternionic Kähler (briefly QK). This condition is equivalent to the statement that the holonomy group of $g$ is contained in $\operatorname{Sp}(\mathrm{n}) \operatorname{Sp}(1)$ [1, 2, 40, 41, 27]. If on a QK manifold there exist an admissible basis $(H)$ such that each almost complex structure $\left(J_{\alpha}\right) \in(H), \alpha=1,2,3$ is parallel with respect to the Levi-Civita connection then the manifold is called hyperKähler (briefly HK). In this case the holonomy group of $g$ is contained in $\operatorname{Sp}(\mathrm{n})$.

The notions of various quaternionic manifolds arise in a natural way from the theory of supersymmetric sigma models as well as in string theory. The geometry of the target space of two-dimensional sigma models with extended supersymmetry is described by the properties of a metric connection with torsion [18, 24]. The geometry of $(4,0)$ supersymmetric two-dimensional sigma models without Wess-Zumino term (torsion) is a hyperKähler manifold. In the presence of torsion the geometry of the target space becomes hyperKähler with torsion (briefly HKT) [25]. This means that the complex structures $J_{\alpha}, \alpha=1,2,3$, are parallel with respect to a metric quaternionic connection with totally skew-symmetric torsion [25]. Local $(4,0)$ supersymmetry requires that the target space of two dimensional sigma models with Wess-Zumino term be either HKT or quaternionic Kähler with torsion (briefly QKT) [33] which means that the quaternionic subbundle is parallel with respect to a metric linear connection with totally skew-symmetric torsion and the torsion 3 -form is of type $(1,2)+(2,1)$ with respect to all almost complex structures in $Q$. The target space of two-dimensional $(4,0)$ supersymmetric sigma models with torsion coupled to $(4,0)$ supergravity is a QKT manifold [26].

HKT spaces with symmetry (homothety) arise in quantum field theories. The geometry coming from the Michelson and Strominger's study of $N=4 B$ supersymmetric quantum mechanics with superconformal $D(2,1, \alpha)$-symmetry is a HKT geometry with a special homothety [32]. These special HKT spaces are studied recently in [38, 39]. It is shown in [39] that the special homothety generate an infinitesimal action of the non-zero quaternions and the quotient space carries a QKT structure which is of instanton type, ie there exists a certain torsion-free quaternionic connection which induces on the real canonical bundle $k^{R}=\Lambda^{4 n} T^{*} M$ a connection with curvature of type $(1,1)$ with respect to each $J_{\alpha}$. Conversely, for a QKT of instanton type it is constructed in [39] a HKT structure with special homothety on the corresponding Swann bundle (a bundle constructed by A. Swann for QK manifold [46]) provided some nondegeneratity (positivity) conditions are fulfilled.

HKT manifolds are also attractive in string theory. The number of preserving supersymmetries in 10-dimensional string theory depends essentially on the number of parallel spinors with respect to a connection with totally skew-symmetric torsion. This imposes restrictions on the holonomy group ie the spinor representation of the holonomy group should have a fixed non-trivial spinor. HKT geometry is one of the possible models since the holonomy group of the HKT-connection is a subgroup of $\operatorname{Sp}(\mathrm{n})$ (see [45, 30, 16, 29, 17] for more precise discussion concerning parallel spinors and holonomy of connection with torsion).

The properties of HKT and QKT geometries resemble those of HK and QK ones, respectively. In particular, HKT 25, 21] and QKT [26] manifolds admit twistor constructions with twistor spaces which have similar properties to those of HK [23] and QK [40, 41, 42, assuming some conditions 
on the torsion 25, 26, 39]. It is shown in [26] that the twistor space of a QKT manifold is always complex manifold provided the dimension is at least 8. Most of the known examples of QKT manifolds are homogeneous constructed in 35] but there are a large class QKT spaces obtained by conformal transformations of QK or HK manifolds [28].

The main object of interest in this article is the differential geometric properties of QKT and HKT manifolds together with their twistor spaces. We find relations between Riemannian scalar curvatures of a QKT space which allows us to express sufficient conditions for a compact 8-dimensional QKT manifold to be QK in terms of its Riemannian scalar curvatures (Theorem 3.7).

We consider two almost complex structures $I_{1}, I_{2}$ on the twistor space $Z$ over a QKT manifold. These structures are originally constructed in [7] and [15] in the QK case, respectively; for QKT $I_{1}$ is investigated in [26] where it is shown that $I_{1}$ is integrable.

We define Riemannian metrics $h_{c}$ on $Z$ depending on a constant $c$ thus obtaining almost hermitian structures on $Z$. Investigating the corresponding almost hermitian geometry we prove that $I_{2}$ is never integrable and the Swann bundle of a QKT manifold admits a HKT structure with special symmetry if and only if $\left(Z, h_{c}, I_{2}\right)$ is a $G_{1}$ manifold according to Gray-Hervella classification [22] (Theorem 5.2). The class of $G_{1}$ manifolds can be viewed as a direct sum of Hermitian and Nearly Kähler manifolds and are characterized by the Nijenhuis tensor to be a 3-form. These manifolds are in particular interest in physics since they arise as a target spaces of $(2,0)$-and $(2,2)$ - supersymmetric sigma models [36]. The physic's data require also the existence of a linear connection $\nabla$ preserving the almost hermitian structure $(g, J)$ and having totally skew-symmetric torsion. The $G_{1}$-manifolds are precisely the object of interest since this is the largest class where such a connection exists [16]. Using the integrability of $I_{1}$ we present new relations between the Ricci forms (Theorem 5.1), the 2-forms which determine the $\mathrm{Sp}(1)$ component of the curvature of the QKT connection.

Our central statement for HKT manifolds is Theorem 6.2 which states that the Lee form is coclosed. This is related with the Gauduchon metric on a compact hermitian manifold stating that on any conformal class there exists a unique metric with co-closed Lee form [19]. As a consequence, we get precise information for the plurigenera of compact HKT manifolds (Theorem 6.4).

Acknowledgements. The final part of the research was done during the visit of S.I. at the Abdus Salam International Centre for Theoretical Physics, Trieste Italy. S.I. thanks the Abdus Salam ICTP for support and the excellent environment.

\section{QKT manifolds}

Let $\mathbf{H}$ be the quaternions and identify $\mathbf{H}^{\mathbf{n}}=\mathbf{R}^{\mathbf{4 n}}$. Let $\mathbf{H}$ acts on the right. This defines an antihomomorphism $\lambda:\{$ unit quaternions $\longrightarrow S O(4 n)$, where $S O(4 n)$ acts on the left. Denote the image by $S p(1)$ and let $I_{0}=\lambda(i), J_{0}=\lambda(j), K_{0}=\lambda(k)$. The Lie algebra of $S p(1)$ is $s p(1)=$ $\operatorname{span}\left\{I_{0}, J_{0}, K_{0}\right\}$. Define $S p(n)=\{A \in S O(4 n): A B=B A$ for all $B \in S p(1)\}$. The Lie algebra of $S p(n)$ is $s p(n)=\{A \in s o(4 n): A B=B A$ for all $B \in s p(1)\}$. Let $S p(n) S p(1)$ be the product of the two groups in $S O(4 n)$. Abstractly, $S p(n) S p(1)=S p(n) \times S p(1) / \mathbf{Z}_{2}$. The Lie algebra of the group $S p(n) S p(1)$ is isomorphic to $s p(n) \oplus s p(1)$.

Let $\left(M, g,\left(J_{\alpha}\right) \in Q, \alpha=1,2,3\right)$ be a 4 n-dimensional almost quaternionic manifold with $Q$ hermitian Riemannian metric $g$ and an admissible basis $\left(J_{\alpha}\right)$. The Kähler form $\Phi_{\alpha}$ of each $J_{\alpha}$ is defined by $\Phi_{\alpha}=g\left(., J_{\alpha}.\right)$. Let $\nabla$ be a quaternionic connection i.e.

$$
\nabla J_{\alpha}=-\omega_{\beta} \otimes J_{\gamma}+\omega_{\gamma} \otimes J_{\beta},
$$

where the $\omega_{\alpha}, \alpha=1,2,3$ are 1 -forms. 
Here and henceforth $(\alpha, \beta, \gamma)$ is a cyclic permutation of $(1,2,3)$.

Let $T(X, Y)=\nabla_{X} Y-\nabla_{Y} X-[X, Y]$ be the torsion tensor of type $(1,2)$ of $\nabla$. We denote by the same letter the torsion tensor of type $(0,3)$ given by $T(X, Y, Z)=g(T(X, Y), Z)$.

An almost quaternionic manifold $\left(M,\left(H_{\alpha}\right) \in \mathcal{Q}\right)$ is $Q K T$ manifold if it admits a hermitian quaternionic structure $(g, \mathcal{Q})$ and a metric quaternionic connection $\nabla$ with totally skew symmetric torsion which is $(1,2)+(2,1)$-form with respect to each $J_{\alpha}, \alpha=1,2,3$ (QKT-connection) ie

$$
T(X, Y, Z)=T\left(J_{\alpha} X, J_{\alpha} Y, Z\right)+T\left(J_{\alpha} X, Y, J_{\alpha} Z\right)+T\left(X, J_{\alpha} Y, J_{\alpha} Z\right) .
$$

It follows that the holonomy group of any QKT-connection is a subgroup of $\operatorname{Sp}(\mathrm{n}) \operatorname{Sp}(1)$ ie the bundle $S O(M)$ of oriented orthonormal frames on a QKT manifold can be reduced to a principal $S p(n) S p(1)$-bundle $P(M)$ and the QKT- connection 1-form on $P(M)$ is $s p(n) \oplus s p(1)$-valued.

Every QKT manifold is a quaternionic manifold [28]. Poon and Swann constructed explicitly a quaternionic torsion-free connection $\nabla^{q}$ on a QKT in [39]. We use their definition of instanton type QKT which requires the curvature of $\nabla^{q}$ on the real canonical bundle to be of type $(1,1)$ with respect to each $J_{\alpha}$. Conversely, any quaternionic manifold locally admits a QKT structure [39]. However, not any quaternionic hermitian structure on a quaternionic manifold is a QKT structure but if a QKT structure exists then it is unique and the torsion 3-form is computed in terms of connection 1-forms $\omega_{\alpha}$ and the exterior derivative of the Kähler forms 28]. One consequence is that a QKT structure persists under conformal transformations of the metric [28]. For HKT, the local existence of HKT structure on any hypercomplex manifold is proved in 21. The uniqueness of a given HKT structure is a consequence of the general results in [20] (see also [21]) which imply that on a hermitian manifold there exists a unique linear connection with totally skew-symmetric torsion preserving the metric and the complex structure, the Bismut connection. This connection was used by Bismut [10] to prove a local index theorem for the Dolbeault operator on non-Kähler manifold. The geometry of this connection is referred to KT-geometry by physicists. Obstructions to the existence of (non-trivial) Dolbeault cohomology groups on a compact KT-manifold are presented in [6, 30].

Ivanov 28] introduced the torsion 1-form on a QKT manifold by the equality

$$
t(X)=\frac{1}{2} \sum_{i=1}^{4 n} T\left(J_{\alpha} X, e_{i}, J_{\alpha} e_{i}\right),
$$

where $\left\{e_{i}\right\}, i=1, \ldots, 4 n$ is an orthonormal basis, and showed that it is independent on $J_{\alpha}$. It turns out that a QKT structure is of instanton type if and only if the exterior differential of the torsion 1 -form $d t$ is of type $(1,1)$ with respect to each $J_{\alpha}$ [39]. We shall call a QKT manifold with zero torsion 1-form balance QKT manifold. The first examples of (compact) balance HKT manifold are constructed by Dotti and Fino [14]. On a compact QKT manifold there always exists in the same conformal class a metric with coclosed torsion 1-form, the Gauduchon metric [28].

\section{Curvature of QKT manifold}

Let $R=[\nabla, \nabla]-\nabla_{[,]}$be the curvature tensor of type $(1,3)$ of $\nabla$. We denote the curvature tensor of type $(0,4) R(X, Y, Z, V)=g(R(X, Y) Z, V)$ by the same letter. There are three Ricci forms and six scalar functions given by $\rho_{\alpha}(X, Y)=\frac{1}{2} \sum_{i=1}^{4 n} R\left(X, Y, e_{i}, J_{\alpha} e_{i}\right), S c a l_{\alpha, \beta}=-\sum_{i=1}^{4 n} \rho_{\alpha}\left(e_{i}, J_{\beta} e_{i}\right)$. The Ricci forms satisfy $n\left[R(X, Y), J_{\alpha}\right]=\rho_{\gamma}(X, Y) J_{\beta}-\rho_{\beta}(X, Y) J_{\gamma}$. The Ricci tensor Ric, the scalar curvatures $S c a l$ and $S c a l_{\alpha}$ of $\nabla$ are defined by $\operatorname{Ric}(X, Y)=\sum_{i=1}^{4 n} R\left(e_{i}, X, Y, e_{i}\right), S c a l=$ 
$\sum_{i=1}^{4 n} \operatorname{Ric}\left(e_{i}, e_{i}\right), S c a l_{\alpha}=-\sum_{i=1}^{4 n} \operatorname{Ric}\left(e_{i}, J_{\alpha} e_{i}\right)$. We shall denote by $R^{g}, \operatorname{Ric}^{g}, \rho_{\alpha}^{g}$, etc. the corresponding object of the metric $g$ ie the same objects taken with respect to the Levi-Civita connection $\nabla^{g}$. We may consider $\left(g, J_{\alpha}\right)$ as an almost hermitian structure. Then the tensor $\rho_{\alpha}^{*}(X, Y)=\rho_{\alpha}^{g}\left(X, J_{\alpha} Y\right)$ is known as the $*$-Ricci tensor of the almost hermitian structure. It is equal to $\rho_{\alpha}^{*}(X, Y)=$ $-\sum_{i=1}^{2 n} R^{g}\left(e_{i}, X, J_{\alpha} Y, J_{\alpha} e_{i}\right)$ by the Bianchi identity. The function $S c a l_{\alpha}^{g}$ is known also as the $*$-scalar curvature. If the $*$-Ricci tensor is a scalar multiple of the metric then the manifold is said to be $*$-Einstein. In general, the $*$-Ricci tensor is not symmetric and the $*$-Einstein condition is a strong condition. We shall see in the last section that the $*$-Ricci tensors of HKT manifold are equal and always symmetric.

A QKT structure is of instanton type if and only if each Ricci form $\rho_{\alpha}$ is of type $(1,1)$ with respect to $J_{\alpha}$ according to 39 .

We show in this section that the scalar functions are not independent and define a new scalar invariant, the 'quaternionic $*$-scalar curvature' on a QKT space. We begin with

Proposition 3.1 Let $\left(M, g,\left(J_{\alpha}\right) \in \mathcal{Q}\right)$ be a 4 n-dimensional $Q K T$ manifold. Then the following identities hold

$$
\begin{gathered}
\sum_{i=1}^{4 n}\left(\nabla_{X} T\right)\left(J_{\alpha} Y, e_{i}, J_{\alpha} e_{i}\right)=2\left(\nabla_{X} t\right) Y \\
\sum_{i, j=1}^{4 n} d T\left(e_{j}, J_{\alpha} e_{j}, e_{i}, J_{\alpha} e_{i}\right)=-8 \delta t+8\|t\|^{2}-\frac{4}{3}\|T\|^{2}, \quad \sum_{i, j=1}^{4 n} d T\left(e_{j}, J_{\beta} e_{j}, e_{i}, J_{\gamma} e_{i}\right)=0 .
\end{gathered}
$$

Proof. The formula (3.4) follows from (2.1) and the definition (2.3) of the torsion 1-form by straightforward calculations. To prove (3.5) we need the following algebraic

Lemma 3.2 For a three form $T$ of type $(1,2)+(2,1)$ with respect to each $J_{\alpha}$ the next equalities hold

$$
\sum_{i, j=1}^{4 n} g\left(T\left(e_{i}, e_{j}\right), T\left(J_{\gamma} e_{i}, J_{\beta} e_{j}\right)\right)=0, \quad \sum_{i, j=1}^{4 n} g\left(T\left(e_{i}, e_{j}\right), T\left(J_{\beta} e_{i}, J_{\beta} e_{j}\right)\right)=\frac{1}{3}\|T\|^{2}
$$

Proof of the Lemma. Put $A=\sum_{i, j=1}^{4 n} g\left(T\left(e_{i}, e_{j}\right), T\left(J_{\gamma} e_{i}, J_{\beta} e_{j}\right)\right)$. Use (2.2) three times to get

$$
\begin{aligned}
A & =\sum_{i, j, k=1}^{4 n} T\left(e_{i}, e_{j}, e_{k}\right)\left(T\left(J_{\gamma} e_{i}, J_{\gamma} e_{j}, J_{\alpha} e_{k}\right)-T\left(J_{\beta} e_{i}, J_{\beta} e_{j}, J_{\alpha} e_{k}\right)\right. \\
A & =\sum_{i, j, k=1}^{4 n} T\left(e_{i}, e_{j}, e_{k}\right)\left(-T\left(J_{\alpha} e_{i}, e_{j}, e_{k}\right)+T\left(J_{\alpha} e_{i}, J_{\beta} e_{j}, J_{\beta} e_{k}\right)\right. \\
A & =\sum_{i, j, k=1}^{4 n} T\left(e_{i}, e_{j}, e_{k}\right)\left(T\left(J_{\alpha} e_{i}, e_{j}, e_{k}\right)-T\left(J_{\gamma} e_{i}, J_{\gamma} e_{j}, J_{\alpha} e_{k}\right)\right.
\end{aligned}
$$

Summing up, we derive $3 A=0$. The proof of the second equality in (3.5) is similar and we omit it.

We need also the expression of $d T$ in terms of $\nabla$ (see e.g. 28, 30, 16]),

$$
\begin{aligned}
& d T(X, Y, Z, U)=\underset{X Y Z}{\sigma}\left\{\left(\nabla_{X} T\right)(Y, Z, U)+g(T(X, Y), T(Z, U)\}\right. \\
& \text { - }\left(\nabla_{U} T\right)(X, Y, Z)+\underset{X Y Z}{\sigma}\{g(T(X, Y), T(Z, U)\},
\end{aligned}
$$

where ${ }_{X Y Z}^{\sigma}$ denote the cyclic sum of $X, Y, Z$. Taking the suitable trace in (3.6) and applying Lemma 3.2, we obtain the first equality in (3.5). We get from(3.6) using (3.4) and Lemma 3.2 that $\sum_{i, j=1}^{4 n} d T\left(e_{j}, J_{\beta} e_{j}, e_{i}, J_{\gamma} e_{i}\right)=-4 \sum_{i, j=1}^{4 n} g\left(T\left(e_{i}, e_{j}\right), T\left(J_{\gamma} e_{i}, J_{\beta} e_{j}\right)\right)=0$.

Q.E.D. 
Proposition 3.3 On a 4 -dimensional $(n>1)$ QKT-manifold we have the equalities:

$$
S c a l_{\alpha, \alpha}=S c a l_{\beta, \beta}=S c a l_{\gamma, \gamma}, \quad S c a l_{\alpha, \beta}=0, \quad \operatorname{Scal}_{\alpha}=\frac{1}{2}\left(d t, \Phi_{\alpha}\right)
$$

Proof. Applying (3.4) consequently to (3.45), (3.33) in [28], we obtain

$$
\begin{aligned}
& (n-1) \rho_{\alpha}\left(X, J_{\alpha} Y\right)=-\frac{n(n-1)}{n+2} \operatorname{Ric}(X, Y)+\frac{n(n-1)}{n+2}\left(\nabla_{X} t\right) Y \\
& +\frac{n}{4(n+2)} \sum_{i=1}^{4 n}\left\{(n+1) d T\left(X, J_{\alpha} Y, e_{i}, J_{\alpha} e_{i}\right)-d T\left(X, J_{\beta} Y, e_{i}, J_{\beta} e_{i}\right)-d T\left(X, J_{\gamma} Y, e_{i}, J_{\gamma} e_{i}\right)\right\} .
\end{aligned}
$$

Take the suitable trace in (3.8), and use (3.5) to get $S c a l_{\alpha, \alpha}=S c a l_{\beta, \beta}, \quad S c a l_{\alpha, \beta}=0$. The last equality in (3.7) is a direct consequence of (3.4), $S c a l_{\alpha, \beta}=0$ and (3.9).

Q.E.D.

Definition. The common trace of the Ricci forms on a $4 \mathrm{n}$-QKT manifold $(n>1)$ we call quaternionic scalar curvature of the $Q K T$ connection and denote it by $S c a l_{Q}, S_{c a l}=S_{Q} l_{\alpha, \alpha}$.

Proposition 3.4 On a 4 -dimensional $(n>1)$ QKT manifold we have

(3.10)Scal ${ }_{\alpha}^{g}=\operatorname{Scal}_{\beta}^{g}=\operatorname{Scal}_{\gamma}^{g}=\operatorname{Scal}_{Q}-\delta t+\|t\|^{2}-\frac{1}{12}\|T\|^{2}, \quad \operatorname{Scal}_{\alpha, \beta}^{g}=\operatorname{Scal}_{\gamma}=\frac{1}{2}\left(d t, \Phi_{\gamma}\right)$.

Proof. We follow 28, 30]. The curvature $R^{g}$ of the Levi-Civita connection is connected with $R$ by

$$
\begin{aligned}
R^{g}(X, Y, Z, U) & \left.\left.=R(X, Y, Z, U)-\frac{1}{2}\left(\nabla_{X} T\right) Y, Z, U\right)+\frac{1}{2}\left(\nabla_{Y} T\right) X, Z, U\right) \\
& -\frac{1}{2} g(T(X, Y), T(Z, U))-\frac{1}{4} g(T(Y, Z), T(X, U))-\frac{1}{4} g(T(Z, X), T(Y, U)) .
\end{aligned}
$$

Taking the traces in (3.11) and using (2.3) we obtain

$$
\begin{aligned}
\rho_{\alpha}^{g}\left(X, J_{\alpha} Y\right) & =\rho_{\alpha}\left(X, J_{\alpha} Y\right)-\frac{1}{2}\left(\nabla_{X} t\right) Y-\frac{1}{2}\left(\nabla_{J_{\alpha} Y} t\right) J_{\alpha} X \\
& +\frac{1}{2} t\left(J_{\alpha} T\left(X, J_{\alpha} Y\right)\right)+\frac{1}{4} \sum_{i=1}^{4 n} g\left(T\left(X, e_{i}\right), T\left(J_{\alpha} Y, J_{\alpha} e_{i}\right)\right),
\end{aligned}
$$

Take the suitable traces in (3.12) and apply Lemma 3.2 and Proposition 3.3 to give (3.10). Q.E.D.

Definition. The common trace of the Riemannian Ricci forms on a 4n-dimensional $(n>1)$ QKT manifold we call quaternionic *-scalar curvature and denote it by $S c a l_{Q}^{g}, S c a l_{Q}^{g}=S c a l_{\alpha}^{g}$.

Proposition 3.5 On a $4 n$-dimensional $(n>1)$ QKT manifold $(M, g, \mathcal{Q})$ the scalar curvatures are related by

$$
\begin{aligned}
S c a l^{g} & =\frac{n+2}{n} S_{c a l}-3 \delta t+2\|t\|^{2}-\frac{1}{12}\|T\|^{2} \\
S c a l_{Q}^{g} & =S c a l_{Q}-\delta t+\|t\|^{2}-\frac{1}{12}\|T\|^{2} \\
S c a l & =\frac{n+2}{n} S_{c a l}-3 \delta t+2\|t\|^{2}-\frac{1}{3}\|T\|^{2} .
\end{aligned}
$$


Proof. We derive from (3.11) that

$$
\begin{gathered}
\operatorname{Ric}^{g}(X, Y)=\operatorname{Ric}(X, Y)+\frac{1}{2} \delta T(X, Y)+\frac{1}{4} \sum_{i=1}^{2 n} g\left(T\left(X, e_{i}\right), T\left(Y, e_{i}\right)\right) \\
\operatorname{Scal}^{g}=S c a l+\frac{1}{4}\|T\|^{2} .
\end{gathered}
$$

Take the trace in (3.9) and use Lemma 3.2 to get the first equality of the proposition.. The second equality is already proved in Proposition 3.10. The last one is a consequence of (3.13) and the already proved first equality in the proposition.

Q.E.D.

As a consequence of the above result, we get

Theorem 3.6 Let $(M, g, \mathcal{Q})$ be a compact 4 n-dimensional $(n>1)$ QKT manifold. Then

$$
\int_{M}\left(S c a l^{g}-S c a l_{Q}^{g}-\frac{2}{n} S c a l_{Q}\right) d V \geq 0 .
$$

The equality in (3.14) is attained if and only if the QKT structure is balance.

$$
\int_{M}\left(S c a l^{g}-2 S c a l_{Q}^{g}-\frac{2-n}{n} S_{c a l}\right) d V \geq 0 .
$$

The equality in (3.15) is attained if and only if the QKT structure is quaternionic Kähler.

Proof. Proposition 3.5 implies $S c a l^{g}-S c a l_{Q}^{g}-\frac{2}{n} S_{c a l} Q=-2 \delta t+2\|t\|^{2}$,

$$
S c a l^{g}-2 S c a l_{Q}^{g}-\frac{2-n}{n} S_{c a l} Q=-\delta t+\frac{1}{12}\|T\|^{2} .
$$

Integrating the last two equalities over $M$ we get the proof.

Q.E.D.

Remark 1. By the conditions of Theorem 3.6 if moreover $g$ is the Gauduchon metric then (3.14) and (3.15) are true without integration over $M$ and the same conclusions are valid.

Applying Theorem 3.6 to an 8-dimensional QKT manifold, put $n=2$ in (3.16), we get the main result of this section

Theorem 3.7 Let $(M, g, \mathcal{Q})$ be an 8-dimensional compact connected QKT manifold. Then

a) $(M, g, \mathcal{Q})$ is a quaternionic Kähler manifold if and only if

$$
\int_{M}\left(S c a l^{g}-2 S c a l_{Q}^{g}\right) d V=0
$$

b) $(M, g, \mathcal{Q})$ is a hyperKähler manifold if and only if the Riemannian scalar curvature and the quaternionic *-scalar curvature both vanish.

In particular, any compact 8-dimensional QKT manifold with flat metric is flat hyperKähler and therefore covered by a hyperKähler torus.

We finishe this section with the following

Theorem 3.8 A 4n-dimensional QKT manifold is of instanton type if and only if each *-Ricci tensor is symmetric. 
Proof. First, we observe that on a QKT manifold $(M, g, \mathcal{Q})$ the $(2,0)+(0,2)$-parts of $\rho_{\alpha}^{g}, \rho_{\alpha}, d t$ with respect to $J_{\alpha}$ are related by the equality

$(3.17) \rho_{\alpha}^{g}\left(X, J_{\alpha} Y\right)+\rho_{\alpha}^{g}\left(J_{\alpha} X, Y\right)=\rho_{\alpha}\left(X, J_{\alpha} Y\right)+\rho_{\alpha}\left(J_{\alpha} X, Y\right)-\frac{1}{2}\left(d t(X, Y)-d t\left(J_{\alpha} X, J_{\alpha} Y\right)\right)$.

Indeed, put $B(X, Y)=\sum_{i=1}^{4 n} g\left(T\left(X, e_{i}\right), T\left(J_{\alpha} Y, J_{\alpha} e_{i}\right)\right)$. The tensor $B$ is symmetric since the $(1,2)+(2,1)$-type property of $T$ leads to the expression

$2 B(X, Y)=\sum_{i, j=1}^{4 n}\left(T\left(X, e_{i}, e_{j}\right), T\left(Y, e_{i}, e_{j}\right)-T\left(X, e_{i}, e_{j}\right), T\left(Y, J_{\alpha} e_{i}, J_{\alpha} e_{j}\right)\right)$ which is clearly symmetric. Then the skew-symmetric part of (3.12) gives (3.17), where we used (2.2) and the equality $d^{\nabla} t(X, Y):=\left(\nabla_{X} t\right) Y-\left(\nabla_{Y} t\right) X=d t(X, Y)-t(T(X, Y))$. Computations in [39] show the identity $\rho_{\alpha}\left(X, J_{\alpha} Y\right)+\rho_{\alpha}\left(J_{\alpha} X, Y\right)=-\frac{n}{2}\left(d t(X, Y)-d t\left(J_{\alpha} X, J_{\alpha} Y\right)\right)$. Consequently, (3.17) gives $\rho_{\alpha}^{g}\left(X, J_{\alpha} Y\right)+\rho_{\alpha}^{g}\left(J_{\alpha} X, Y\right)=-\frac{n+1}{2}\left(d t(X, Y)-d t\left(J_{\alpha} X, J_{\alpha} Y\right)\right)$. Hence, $M$ is of instanton type if and only if $\rho_{\alpha}^{g}$ is of type $(1,1)$ with respect to $J_{\alpha}$. The latter property is equivalent to the condition that the corresponding $*$-Ricci tensor $\rho_{\alpha}^{*}$ is symmetric.

Q.E.D.

\section{Twistor space of QKT manifolds}

Keeping in mind the presence of torsion, the setup below is similar to that of [44, 12] and very closed to that of [5].

Let $(M, g)$ be a $4 n$-dimensional QKT manifold and $\pi: P(M) \longrightarrow M$ be the natural projection. For each $u \in P(M)$ we consider the linear isomorphism $j(u)$ on $T_{\pi(u)} M$ defined by $j(u)=u J_{0} u^{-1}$. It is easy to see that $j(u)^{2}=-i d$ and $g(j(u) X, j(u) Y)=g(X, Y)$ for all $X, Y \in T_{\pi(u)} M$, i.e. $j(u)$ is an orthogonal complex structure at $\pi(u)$. For each point $p \in M$ we define $Z_{p}(M)=\{j(u): u \in$ $P(M), \pi(u)=p\}$. In other words, $Z_{p}(M)$ is the space of all orthogonal complex structures in the tangent space $T_{p} M$ which are compatible with the QKT structure.

We put $Z=\bigcup_{p \in M} Z_{p}(M)$. Let $H=S p(n) S p(1) \cap U(2 n)$. There is a bijective correspondence between the symmetric space $S p(n) S p(1) / H=S p(1) / U(1)=\mathbf{C P}^{\mathbf{1}}=\mathbf{S}^{\mathbf{2}}$ and $Z_{p}(M)$ for every $p \in M$. So we can consider $Z$ as the associated fibre bundle of $P(M)$ with standard fibre $S p(n) S p(1) / H=\mathbf{C P}^{\mathbf{1}}$. Hence, $P(M)$ is a principal fibre bundle over $Z$ with structure group $H$ and projection $j$. If $\pi_{1}: Z \longrightarrow M$ is the projection, we have that $\pi_{1} \circ j=\pi$. We consider the symmetric space $S p(n) S p(1) / H$. We have the following Cartan decomposition $s p(n) \oplus s p(1)=h \oplus m$, where $h=\left\{A \in s p(n) \oplus s p(1): A J_{0}=J_{0} A\right\}=(s p(n) \oplus s p(1)) \cap u(2 n)$ is the Lie algebra of $H$ and $m=\left\{A \in \operatorname{sp}(n) \oplus s p(1): A J_{0}=-J_{0} A\right\}$. It is clear that $m$ is generated by $I_{0}$ and $K_{0}$, i.e. $m=\operatorname{span}\left\{I_{0}, K_{0}\right\}$. Hence, if $A \in m$ then $J_{0} A \in m$. Let $($,$) be the inner product in g l(4 n, \mathbf{R})$ defined by $(A, B)=\operatorname{trace}\left(A B^{t}\right)=\sum_{i=1}^{4 n}<A e_{i}, B e_{i}>$ for $A, B \in \operatorname{gl}(4 n, \mathbf{R})$, where $<,>$ is the canonical inner product in $\mathbf{R}^{\mathbf{4 n}}$. It is clear that $\operatorname{sp}(n) \perp s p(1)$ and $I_{0}, J_{0}, K_{0}$ form an orthogonal basis of $s p(1)$ with $\left(I_{0}, I_{0}\right)=\left(J_{0}, J_{0}\right)=\left(K_{0}, K_{0}\right)=4 n$. Hence, $h \perp m$.

Let $u \in P(M)$ and $Q_{u}$ is the horizontal subspace of the tangent space $T_{u} P(M)$ induced by the QKT- connection on $\mathrm{M}$ ([31]). The vertical space is $h_{u}^{*} \oplus m_{u}^{*}$, where $h_{u}^{*}=\left\{A_{u}^{*}: A \in\right.$ $h\}, m_{u}^{*}=\left\{A_{u}^{*}: A \in m\right\}$. Hence, $T_{u} P(M)=h_{u}^{*} \oplus m_{u}^{*} \oplus Q_{u}$. For each $u \in P(M)$ we put $V_{j(u)}=j_{* u}\left(h_{u}^{*} \oplus m_{u}^{*}\right), H_{j(u)}=j_{* u} Q_{u}$. Thus we obtain the vertical and horizontal distributions $V$ and $H$ on $Z$. Since $P(M)$ is a principal fibre bundle over $Z$ with structure group $H$ we have

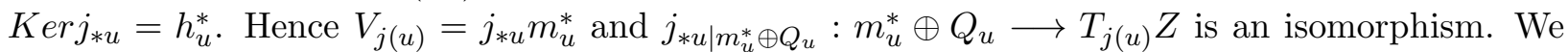
define almost complex structures $I_{1}$ and $I_{2}$ on $Z$ by

$$
\begin{aligned}
& J_{1} j_{* u} A^{*}=j_{* u}\left(J_{0} A\right)^{*}, \quad J_{2} j_{* u} A^{*}=-j_{* u}\left(J_{0} A\right)^{*} \\
& I_{i} j_{* u} B(\xi)=j_{* u} B\left(J_{0} \xi\right), \quad i=1,2,
\end{aligned}
$$


for $u \in P(M), A \in m, \xi \in \mathbf{R}^{4 \mathbf{n}}$. For twistor bundles of 4-manifolds the almost complex structure $I_{1}$ is introduced in [7] and the almost complex structure $I_{2}$ is introduced in [15] by use of the horizontal spaces of the Levi-Civita connection. The almost complex structure $I_{1}$ for QK, HKT and QKT manifolds was constructed in [40, 25, 26], respectively, where it is proved that it is actually integrable. For every $c>0$ a Riemannian metric $h_{c}$ on $\mathrm{Z}$ is defined by

$$
\begin{aligned}
& h_{c}\left(j_{* u} A^{*}, j_{* u} B^{*}\right)=c^{2}(A, B), \quad h_{c}\left(j_{* u} A^{*}, j_{* u} B(\xi)\right)=0 \\
& h_{c}\left(j_{* u} B(\xi), j_{* u} B(\eta)\right)=<\xi, \eta>,
\end{aligned}
$$

for $u \in P(M), A, B \in m, \xi, \eta \in \mathbf{R}^{\mathbf{4 n}}$.

It is clear that $\left(I_{i}, h_{c}\right), i=1,2$, determine two families of almost Hermitian structures on $Z$.

In the QK case the properties of the Almost hermitian geometry of $\left(I_{i}, h_{c}\right), i=1,2$ are considered in [13, 5]. Below we follow [5] keeping in mind the presence of torsion.

We split the curvature of a QKT-connection into $s p(n)$-valued part $R^{\prime}$ and $s p(1)$-valued part $R^{\prime \prime}$ following the classical scheme (see e.g. [3, 27, 9])

Proposition 4.1 The curvature of a QKT manifold splits as follows

$$
\begin{aligned}
R(X, Y)= & R^{\prime}(X, Y)+\frac{1}{2 n}\left(\rho_{1}(X, Y) J_{1}+\rho_{2}(X, Y) J_{2}+\rho_{3}(X, Y) J_{3}\right) \\
& {\left[R^{\prime}(X, Y), J_{\alpha}\right]=0, \quad \alpha=1,2,3 . }
\end{aligned}
$$

We denote by $A^{*}$ (resp. $B(\xi)$ ) the fundamental vector field (resp. the standard horizontal vector field) on $P(M)$ corresponding to $A \in s p(n) \oplus s p(1)$ (resp. $\xi \in \mathbf{R}^{\mathbf{4 n}}$ ). Let $\Omega, \Theta$ be the curvature 2-form and the torsion 2-form for the QKT-connection on $P(M)$, respectively ([31] ).

We shall denote the splitting of the $s p(n) \oplus s p(1)$-valued curvature 2-form $\Omega$ on $P(M)$, corresponding to Proposition 4.1, by $\Omega=\Omega^{\prime}+\Omega^{\prime \prime}$, where $\Omega^{\prime}$ is a $s p(n)$-valued 2 -form and $\Omega^{\prime \prime}$ is a $s p(1)$-valued form. Explicitly, we have $\Omega^{\prime \prime}=\Omega_{1}^{\prime \prime} I_{0}+\Omega_{2}^{\prime \prime} J_{0}+\Omega_{3}^{\prime \prime} K_{0}$, where $\Omega_{\alpha}^{\prime \prime}, \alpha=1,2,3$, are 2 -forms. If $\xi, \eta, \zeta \in \mathbf{R}^{\mathbf{4 n}}$, then the 2 -forms $\Omega_{\alpha}^{\prime \prime}, \alpha=1,2,3$, are given by

$$
\Omega_{\alpha}^{\prime \prime}(B(\xi), B(\eta))=\frac{1}{2 n} \rho_{\alpha}(X, Y), \quad X=u(\xi), Y=u(\eta) .
$$

Since $T$ is 3 -form of type $(1,2)+(2,1)$, the torsion 2 -form $\Theta$ has the properties

$$
\begin{aligned}
& <\Theta_{u}(B(\xi), B(\eta)), \zeta>=-<\Theta_{u}(B(\xi), B(\zeta)), \eta> \\
& <\Theta_{u}(B(\xi), B(\eta)), \zeta>=<\Theta_{u}\left(B\left(J_{0} \xi\right), B\left(J_{0} \eta\right)\right), \zeta> \\
& +<\Theta_{u}\left(B\left(J_{0} \xi\right), B(\eta)\right), J_{0} \zeta>+<\Theta_{u}\left(B(\xi), B\left(J_{0} \eta\right)\right), J_{0} \zeta>
\end{aligned}
$$

Let $F_{i}(X, Y, Z)=h_{c}\left(\left(D_{X} I_{i}\right) Y, Z\right), i=1,2$, where $D$ is the covariant derivative of the Levi-Civita connection of $h_{c}$. We denote by $\mathrm{K}$ the curvature tensor of $h_{c}$.

In the rest of the paper $A, B, C, D \in m, \xi, \eta, \zeta, \tau \in \mathbf{R}^{4 n}$.

The calculations made in [5] for the twistor space over QK manifold can be performed in our case by taking into account the torsion and their properties. In this way, using (4.18), (4.19) and (4.21), we obtain our technical tools, namely

Proposition 4.2 The next equalities hold at $u \in P(M)$ :

$$
F_{1,2}\left(j_{* u} A^{*}, j_{* u} B^{*}, j_{* u} C^{*}\right)=0, \quad F_{1,2}\left(j_{* u} A^{*}, j_{* u} B^{*}, j_{* u} B(\xi)\right)=0,
$$




$$
\begin{aligned}
F_{1,2}\left(j_{* u} A^{*}, j_{* u} B(\xi), j_{* u} B(\eta)\right)= & \frac{c^{2}}{2}\left(A, \Omega\left(B\left(J_{0} \xi\right), B(\eta)\right)+\right. \\
& +\frac{c^{2}}{2}\left(A, \Omega\left(B(\xi), B\left(J_{0} \eta\right)\right)+2<J_{0} \xi, \eta>,\right.
\end{aligned}
$$

(4.22) $F_{1,2}\left(j_{* u} B(\xi), j_{* u} A^{*}, j_{* u} B^{*}\right)=0$,

$$
\begin{aligned}
& F_{2}\left(j_{* u} B(\xi), j_{* u} A^{*}, j_{* u} B(\eta)\right)=\frac{c^{2}}{2}\left(A, J_{0} \Omega(B(\xi), B(\eta))+\frac{c^{2}}{2}\left(A, \Omega\left(B(\xi), B\left(J_{0} \eta\right)\right),\right.\right. \\
& F_{1}\left(j_{* u} B(\xi), j_{* u} A^{*}, j_{* u} B(\eta)\right)=\frac{c^{2}}{2}\left(J_{0} A, \Omega(B(\xi), B(\eta))+\frac{c^{2}}{2}\left(A, \Omega\left(B(\xi), B\left(J_{0} \eta\right)\right),\right.\right. \\
& F_{1,2}\left(j_{* u} B(\xi), j_{* u} B(\eta), j_{* u} B(\zeta)\right)=-\frac{1}{2}<\Theta\left(B(\xi), B\left(J_{0} \eta\right)\right), \zeta>-\frac{1}{2}<\Theta(B(\xi), B(\eta)), J_{0} \zeta>
\end{aligned}
$$

For the curvature tensor $K$ we have

Proposition 4.3 The the following equalities hold at any $u \in P(M)$ :

$$
\begin{aligned}
& K\left(j_{* u} A^{*}, j_{* u} B^{*}, j_{* u} C^{*}, j_{* u} D^{*}\right)=-c^{2}([A, B],[C, D]), \\
& K\left(j_{* u} A^{*}, j_{* u} B^{*}, j_{* u} C^{*}, j_{* u} B(\xi)\right)=0, \\
& K\left(j_{* u} A^{*}, j_{* u} B(\xi), j_{* u} B^{*}, j_{* u} B(\eta)\right)=\frac{c^{2}}{2}([A, B], \Omega(B(\xi), B(\eta))- \\
& \quad-\frac{c^{4}}{4}\left(B, \Omega\left(B(\xi), B\left(e_{i}\right)\right)(u)\right)\left(A, \Omega\left(B(\eta), B\left(e_{i}\right)\right),\right. \\
& K\left(j_{* u} B(\xi), j_{* u} B(\eta), j_{* u} B(\zeta), j_{* u} A^{*}\right)=\frac{c^{2}}{2}(A, B(\zeta) \Omega(B(\xi), B(\eta))) \\
& \quad-\frac{c^{2}}{4}(A, \Omega([B(\eta), B(\zeta)], B(\xi)))-\frac{c^{2}}{4}(A, \Omega([B(\zeta), B(\xi)], B(\eta))), \\
& K\left(j_{* u} B(\xi), j_{* u} B(\eta), j_{* u} B(\zeta), j_{* u} B(\tau)\right)=<\Omega(B(\xi), B(\eta)) \zeta, \tau> \\
& \quad-\frac{c^{2}}{4}\left(\Omega_{m}(B(\xi), B(\tau)), \Omega_{m}(B(\eta), B(\zeta))\right)+\frac{c^{2}}{4}\left(\Omega_{m}(B(\xi), B(\zeta)), \Omega_{m}(B(\eta), B(\tau))\right) \\
& \quad+\frac{c^{2}}{2}\left(\Omega_{m}(B(\xi), B(\eta)), \Omega_{m}(B(\zeta), B(\tau))\right)-\frac{1}{4}<\Theta(B(\xi), B(\tau)), \Theta(B(\eta), B(\zeta))> \\
& \quad+\frac{1}{4}<\Theta(B(\xi), B(\zeta)), \Theta(B(\eta), B(\tau))>-\frac{1}{2}<\Theta(B(\xi), B(\eta)), \Theta(B(\zeta), B(\tau))> \\
& \quad-\frac{1}{2}<B(\xi) \Theta(B(\eta), B(\zeta)), \tau>+\frac{1}{2}<B(\eta) \Theta(B(\xi), B(\zeta)), \tau>,
\end{aligned}
$$

where $\Omega_{m}$ denotes the $m$-component of $\Omega$.

\section{Almost Hermitian geometry of $\left(Z, h_{c}, I_{i}\right)$}

Let $(M, g, J)$ be a $2 n$-dimensional almost Hermitian manifold and let $F(X, Y, Z)=g\left(\left(\nabla_{X}^{g} J\right) Y, Z\right)$, where $\nabla^{g}$ is the covariant differentiation of the Levi-Civita connection on $M$. We recall the definition of some classes according to the Gray-Hervella classification [22] in terms of the notations we use: $(M, g, J)$ is Kähler if $F=0$; Hermitian if $H(X, Y, Z)=F(X, Y, Z)-F(J X, J Y, Z)=0$; semi-Kähler if $\operatorname{tr} F=0$, quasi-Kähler if $F(X, Y, Z)+F(J X, J Y, Z)=0$; nearly Kähler if $F(X, Y, Z)+F(Y, X, Z)=0 ; \quad$ almost Kähler if $F(X, Y, Z)+F(Y, Z, X)+F(Z, X, Y)=0 ; G_{1}$ manifold if $\psi(X, Y, Z)=F(X, Y, Z)+F(Y, X, Z)-F(J X, J Y, Z)-F(J Y, J X, Z)=0$. 
Theorem 5.1 On a QKT mamnifold $\left(M^{4 n}, g,\left(J_{\alpha}\right) \in \mathcal{Q}\right)$ the (2,0)+(0,2)-parts of the Ricci forms $\rho_{\alpha}, \rho_{\beta}$ with respect to $J_{\gamma}$ coincide.

Proof. We claim the following identities hold

$$
\rho_{\alpha}\left(J_{\beta} X, J_{\beta} Y\right)-\rho_{\alpha}(X, Y)=\rho_{\gamma}\left(J_{\beta} X, Y\right)+\rho_{\gamma}\left(X, J_{\beta} Y\right)
$$

Indeed, consider the almost hermitian structure $\left(h_{c}, I_{1}\right)$ on the twistor space $Z$. The almost complex structure $I_{1}$ is integrable [26]. Therefore $\left(Z, h_{c}, I_{1}\right)$ is a Hermitian manifold. We calculate taking into account (4.22) that

$$
\begin{aligned}
& 0=\frac{2}{c^{2}}\left(F_{1}\left(j_{* u} A^{*}, j_{* u} B(\xi), j_{* u} B(\eta)\right)-F_{1}\left(I_{1}\left(j_{* u} A^{*}\right), I_{1}\left(j_{* u} B(\xi)\right), j_{* u} B(\eta)\right)\right)= \\
& \left(J_{0} A, \Omega(B(\xi), B(\eta))+\left(A, \Omega\left(B\left(J_{0} \xi\right), B(\eta)\right)+\left(A, \Omega\left(B(\xi), B\left(J_{0} \eta\right)\right)-\left(J_{0} A, \Omega\left(B\left(J_{0} \xi\right), B\left(J_{0} \eta\right)\right) .\right.\right.\right.\right.
\end{aligned}
$$

The $m$ component $\Omega_{m}$ is given by $2 n \Omega_{m}(B(\xi), B(\eta))=\rho_{1}(B(\xi), B(\eta)) I_{0}+\rho_{3}(B(\xi), B(\eta)) K_{0}$. The last two equalities imply

$$
\rho_{1}\left(B\left(J_{0} \xi\right), B(\eta)\right)+\rho_{1}\left(B(\xi), B\left(J_{0} \eta\right)\right)=\rho_{3}\left(B\left(J_{0} \xi\right), B\left(J_{0} \eta\right)\right)-\rho_{3}(B(\xi), B(\eta))
$$

The proof is completed by puting $J_{1}=I_{0}, J_{2}=J_{0}, J_{3}=K_{0}$.

Q.E.D.

We recall the notion of Swann bundle [46, 37, 39]. On a 4n dimensional QKT manifold $M$ it is defined by $\mathcal{U}(M)=P \times_{S p(n) S p(1)} H^{*} /\{ \pm\}$, where $H^{*}$ are the nonzero quaternions. It carries a hypercomplex structure [46, 37, 39]. If $M$ is of instanton type and the condition

$$
\rho_{\alpha}\left(J_{\alpha} X, Y\right)+\rho_{\alpha}\left(J_{\gamma} X, J_{\beta} Y\right)=\frac{1}{c^{2}} g(X, Y)
$$

holds then $\mathcal{U}(M)$ has a HKT structure with special homothety [39]. This generalize the Swann result stating that if $M$ is QK then $\mathcal{U}(M)$ carries HK structure 46]. Another construction of HK structure on the Swann bundle over QKT is presented in [26] by the assumption that $d T$ is a $(2,2)$-form with respect to each $J_{\alpha}$.

Theorem 5.2 Let $\left(M^{4 n}, g,\left(J_{\alpha}\right) \in \mathcal{Q}\right)$ be a $Q K T$ manifold with twistor space $Z$.

a) The almost complex structure $I_{2}$ on $Z$ is never integrable.

b) The space $\left(Z, h_{c}, I_{2}\right)$ is a $G_{1}$ manifold if and only if the Swann bundle admits HKT structure with special homothety.

c) The spaces $\left(Z, h_{c}, I_{i}\right), \quad i=1,2$ are semi-Kähler manifold if and only if the QKT structure is balance, $t=0$.

d) If $\left(Z, h_{c}, I_{2}\right)$ is quasiKähler, almost Kähler, nearly Kähler or $\left(Z, h_{c}, I_{1}\right)$ is Kähler then the torsion is zero and $M$ is a $Q K$ manifold.

Proof. The almost complex structure $I_{2}$ is integrable if and only if $H=0$. We obtain using (4.22) that

$$
\begin{aligned}
H\left(j_{* u} B(\xi), j_{* u} A^{*}, j_{* u} B(\eta)\right)= & \frac{c^{2}}{2}\left(J_{0} A, \Omega\left(B\left(J_{0} \xi\right), B\left(J_{0} \eta\right)\right)\right)-\frac{c^{2}}{2}\left(J_{0} A, \Omega(B(\xi), B(\eta))\right) \\
& \left.+\frac{c^{2}}{2}\left(A, \Omega\left(B\left(J_{0} \xi\right), B(\eta)\right)\right)+\frac{c^{2}}{2} A, \Omega\left(B(\xi), B\left(J_{0} \eta\right)\right)\right) \\
H\left(j_{* u} A^{*}, j_{* u} B(\xi), j_{* u} B(\eta)\right)= & 4<A J_{0} \xi, \eta>+H\left(j_{* u} B(\xi), j_{* u} A^{*}, j_{* u} B(\eta)\right)
\end{aligned}
$$


Hence, $<A J_{0} \xi, \eta>=0$ which is impossible.

For b), $\left(Z, h_{c}, I_{2}\right)$ is a $G_{1}$-manifold if and only if $\psi=0$. We get by (4.22) that the only non-zero term of $\psi$ is

$$
\begin{aligned}
& \psi\left(j_{* u} A^{*}, j_{* u} B(\xi), j_{* u} B(\eta)\right)=4<A J_{0} \xi, \eta>-c^{2}\left(J_{0} A, \Omega(B(\xi), B(\eta))\right)+ \\
& c^{2}\left(A, \Omega\left(B\left(J_{0} \xi\right), B(\eta)\right)\right)+c^{2}\left(A, \Omega\left(B(\xi), B\left(J_{0} \eta\right)\right)\right)+c^{2}\left(J_{0} A, \Omega\left(B\left(J_{0} \xi\right), B\left(J_{0} \eta\right)\right)\right)
\end{aligned}
$$

The last equality is equivalent to

$$
\begin{aligned}
& c^{2} \rho_{1}\left(B\left(J_{0} \xi\right), B(\eta)\right)+c^{2} \rho_{1}\left(B(\xi), B\left(J_{0} \eta\right)\right)=-2<K_{0} \xi, \eta> \\
& c^{2} \rho_{3}\left(B\left(J_{0} \xi\right), B(\eta)\right)+c^{2} \rho_{3}\left(B(\xi), B\left(J_{0} \eta\right)\right)=2<I_{0} \xi, \eta>
\end{aligned}
$$

Put $J_{1}=I_{0}, J_{2}=J_{0}, J_{3}=K_{0}$ in $(5.26)$ we derive $(5.25)$. Combining the already proved (5.25) with (5.24) we get that $\rho_{\alpha}$ is of type $(1,1)$ with respect to $J_{\alpha}$. Hence, the QKT structure is of instanton type. The rest of b) follows by Theorem 6.1 and Remark 6.3 in [39].

We use (4.22) again to prove $\mathrm{c})$. We have $0=\operatorname{tr} F_{i}\left(j_{* u} A^{*}\right)=-\frac{c^{2}}{2} \sum_{k=1}^{4 n}\left(A, \Omega\left(B\left(e_{k}\right), B\left(J_{0} e_{k}\right)\right)\right)$ which is equivalent to $\sum_{k=1}^{4 n} \rho_{\alpha}\left(e_{k}, J_{\beta} e_{k}\right)=0$ by Proposition 3.1. Further, $0=\operatorname{tr} F_{i}\left(j_{* u} B(\xi)\right)=$ $-\frac{1}{2} \sum_{k=1}^{4 n}<\Theta\left(B\left(e_{k}\right), B\left(J_{0} e_{k}\right)\right), \xi>=t(u(\xi))$.

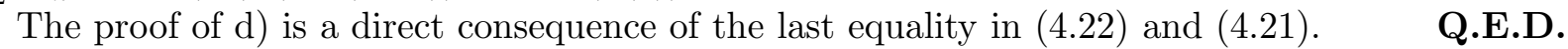

Remark 2. We may consider the twistor space of an Almost quaternionic manifold and construct the almost complex structure $I_{1}$ using horizontal spaces of a quaternionic connection with skew-symmetric torsion. It follows that $I_{1}$ is integrable if and only if the torsion is $(1,2)+(2,1)$-form ie it is a QKT manifold.

Examples. The twistor space $\left(Z, h_{c}, I_{i}\right), i=1,2$ of balance HKT structures on the nilpotent Lie groups constructed in [14] is semi-Kähler for $I_{2}$ and hermitian semi-Kähler (balance) for $I_{1}$.

\section{Geometry of HKT manifold and twistor construction}

We recall some notations. The lee form $\theta$ of a 2 n-dimensional almost Hermitian manifold $(M, g, J)$ with Kähler form $\Phi=g(., J$.$) is defined by \theta=-\delta \Phi \circ J$. On a HKT manifold there are three Lee forms corresponding to $J_{\alpha}, \alpha=1,2,3$ which are equal. The common Lee form $\theta$ is called the Lee form of the HKT structure. It turns out that the Lee form of a HKT manifold is equal to the torsion 1-form, $\theta=t[30,28]$.

On a HKT manifold all the Ricci forms vanish and the exterior differential of the Lee form is of type $(1,1)$ with respect to each $J_{\alpha}[6,30]$. The latter property can be easily seen comparing the curvatures of the Bismut and Chern connection taken with respect to any hermitian structure $J_{\alpha}$ on the corresponding canonical bundle. They differ by $d\left(J_{\alpha} \theta\right)$, where $J_{\alpha} \theta(X)=-\theta\left(J_{\alpha} X\right)$. The curvature of the Chern connection is of type $(1,1)$, the curvature of Bismut connection vanishes and the $(2,0)+(0,2)$-parts of $d \theta$ and $d\left(J_{\alpha} \theta\right)$ coincide. Thus, Theorem 3.8 implies that on a HKT manifold the Riemannian Ricci form $\rho_{\alpha}^{g}$ is of type $(1,1)$ with respect to the complex structure $J_{\alpha}$ and therefore the $*$-Ricci tensors are symmetric. In fact, we derive more using the twistor construction.

Proposition 6.1 Let $\left(Z, h_{c}, I_{i}, i=1,2\right)$ be a twistor space of a 4 n dimensional $(n>1) H K T$ manifold $\left(M, g,\left(J_{\alpha}\right)\right)$ and $X=u(\xi), Y=u(\eta) \in T_{\pi(u)} M$. The Ricci tensor $\rho$ and the $*$-Ricci tensor $\rho_{i}^{*}$ for $\left(Z, h_{c}, I_{i}\right), i=1,2$ are given by

$$
\operatorname{Ric}\left(j_{* u} A^{*}, j_{* u} B^{*}\right)=\rho_{I_{i}}^{h_{c}}\left(j_{* u} A^{*}, j_{* u} B^{*}\right)=\frac{1}{n c^{2}} h_{c}\left(j_{* u} A^{*}, j_{* u} B^{*}\right), \quad i=1,2
$$




$$
\begin{aligned}
& \operatorname{Ric}\left(j_{* u} B(\xi), j_{* u} A^{*}\right)=\rho_{I_{i}}^{h_{c}}\left(j_{* u} B(\xi), j_{* u} A^{*}\right)=0, \quad i=1,2 ; \\
& \operatorname{Ric}\left(j_{* u} B(\xi), j_{* u} B(\eta)\right)=\operatorname{Ric}(X, Y) \\
& \rho_{I_{i}}^{h_{c}}\left(j_{* u} B(\xi), j_{* u} B(\eta)\right)=\rho_{\alpha}^{*}(X, Y), \quad i=1,2, \quad \alpha=1,2,3 .
\end{aligned}
$$

In particular, the $*$-Ricci tensors on a HKT manifold are all equal and symmetric.

If the HKT space is Einstein (resp. *-Einstein with respect to some $J_{\alpha}$ ) with positive scalar curvature $S_{c a l}^{g}$ (resp. Scal $Q_{Q}^{g}$ ) then there exists an Einstein hermitian structure $\left(Z, h_{c}, I_{1}\right), c^{2}=$ $\frac{4}{\text { Scalg }}$ (resp. *-Einstein almost hermitian structure $\left(Z, h_{c}, I_{2}\right), c^{2}=\frac{4}{\text { Scal }_{Q}^{g}}$ ).

Proof. Put $J_{0}=J_{\alpha}$ at $p=\pi(u)$. Take the trace into (4.23) and compare the result with (3.13), (3.12) to get the formulas in the theorem. Further, the left hand side of all formulas in the proposition are independent on the particular choice of $J_{0}$. Hence, the $*$-Rici tensors are equal and symmetric. The formula for the constant $c^{2}$ is a consequence of the fact that the $*$-Einstein curvature is exactly equal to the quaternionic curvature by Proposition 3.4.

Q.E.D.

Remark 3. In view of the above result, the $*$-Einstein condition on a HKT manifold does not imposses restrictions on the $(2,0)+(0,2)$-part of the $*$-Ricci tensor.

The next theorem is our main result concerning HKT geometry

Theorem 6.2 On a $4 n$-dimensional $(n>1)$ HKT manifold the Lee form is coclosed, $\theta=0$.

a) $\mathrm{Scal}^{g}-\mathrm{Scal}_{Q}^{g} \geq 0$ with the equality if and only if the HKT structure is balance;

b) $S_{c a l}^{g}-2 S c a l_{Q}^{g} \geq 0$ with the equality if and only if the HKT structure is hyperKähler;

In particular, any HKT structure with flat metric is hyperKähler.

Proof. We shall prove first the following

Lemma 6.3 On any $4 n$-dimensional $(n>1)$ HKT manifold the next equality holds

$$
\sum_{i=1}^{4 n}\left(\nabla_{e_{i}} T\right)\left(X, Y, J_{\alpha} e_{i}\right)=0, \quad \alpha=1,2,3 .
$$

Proof of the Lemma. We use the twistor construction. We compute the $*$-Ricci tensor of $\left(Z, h_{c}, I_{1}\right)$ in two ways and compare the results to get the assertion. On one hand, we have already done it in the proof of Proposition 6.1, namely $\rho_{I_{1}}^{h_{c}}\left(j_{* u}\left(j_{* u} B(\xi), j_{* u} B(\eta)\right)=\rho_{\alpha}^{g}\left(X, J_{\alpha} Y\right)\right.$, where $\rho_{\alpha}^{g}\left(X, J_{\alpha} Y\right)$ is given by (3.12). On the other hand, using (4.23), we derive

$$
\begin{aligned}
& \rho_{I_{1}}^{h_{c}}\left(j_{* u} B(\xi), j_{* u} B(\eta)\right)= \\
& -\operatorname{Ric}(X, Y)-\frac{3}{4} \sum_{i=1}^{4 n} g\left(T\left(X, e_{i}\right), T\left(J_{\alpha} Y, J_{\alpha} e_{i}\right)+\left(\nabla_{X} \theta\right) Y-\frac{1}{2} \theta\left(J_{\alpha} T\left(X, J_{\alpha} Y\right)\right) .\right.
\end{aligned}
$$

On a HKT manifold $d T$ is $(2,2)$-form with respect to each complex structure $J_{\alpha}$ and therefore all terms in the second line in (4.23) are equal (see [28]). Thus, (4.23) gives

$$
\operatorname{Ric}(X, Y)=\left(\nabla_{X} \theta\right) Y+\frac{1}{4} \sum_{i=1}^{4 n} d T\left(X, J_{\alpha} Y, e_{i}, J_{\alpha} e_{i}\right) .
$$

Substituting (6.29) into (6.28), we obtain

$$
\begin{aligned}
& \rho_{I_{1}}^{h_{c}}\left(j_{* u} B(\xi), j_{* u} B(\eta)\right)= \\
& -\frac{1}{4} \sum_{i=1}^{4 n} d T\left(X, J_{\alpha} Y, e_{i}, J_{\alpha} e_{i}\right)-\frac{3}{4} \sum_{i=1}^{4 n} g\left(T\left(X, e_{i}\right), T\left(J_{\alpha} Y, J_{\alpha} e_{i}\right)-\frac{1}{2} \theta\left(J_{\alpha} T\left(X, J_{\alpha} Y\right) .\right.\right.
\end{aligned}
$$


Take the trace in (3.6) keeping in mind Proposition 3.1 and (2.3), substitute the result into (6.30) and compare the obtained equality with $\rho_{\alpha}^{g}\left(X, J_{\alpha} Y\right)$ given by (3.12) to get the proof of the Lemma.

Further, the trace in $(6.27)$ leads to $0=\sum_{i=1}^{4 n}\left(\nabla_{e_{i}} \theta\right) e_{i}=\sum_{i=1}^{4 n}\left(\nabla_{e_{i}}^{g} \theta\right) e_{i}=-\delta \theta$ since $T$ is totallyskew symmetric. The rest of the theorem follows by Proposition 3.5 as in the proof of Theorem 3.6. Q.E.D.

Remark 4. As a consequence of $(6.29)$ we get that on a 4 n dimensional $(n \geq 2)$ HKT manifold the Ricci tensor is symmetric if and only if $d^{\nabla} \theta(X, Y)=0$. In particular, on a balance $4 n$ dimensional $(n \geq 2)$ HKT manifold the Ricci tensor is $J_{\alpha}$-invariant and symmetric. Therefore, the torsion 3-form is coclosed, $\delta T=0$. We note that this is true in more general situation on any balance Hermitian manifold which Bismut connection has holonomy contained in the special unitary group SU [30].

As an application, we are able to improve the Gauduchon plurigenera theorem for HKT manifold (see [19, 6, 30]). Recall that for $m>0$ the $m-t h$ plurigenus of a compact complex manifold $(M, J)$ is defined by $p_{m}(J)=\operatorname{dim} H^{0}\left(M, \mathcal{O}\left(K^{m}\right)\right)$.

Theorem 6.4 Let $\left(M, g,\left(J_{\alpha}\right)\right)$ be a compact $4 n$-dimensional $(n>1)$ HKT manifold. Then

a) $p_{m}\left(J_{\alpha}\right)=0$ if and only if the HKT structure is not balance, $\theta \neq 0$;

b) $p_{m}\left(J_{\alpha}\right)=1$ if and only if the HKT structure is balance, $\theta=0$

\section{References}

[1] D.V.Alekseevsky, Riemannian spaces with exceptional holonomy groups, Funnkcional. Anal. Prilozen. 2 (1968), 1-10.

[2] D.V.Alekseevsky, Compact quaternion spaces, Funnkcional. Anal. Prilozen. 2 (1968), 11-20.

[3] D.V.Alekseevsky, S.Marchiafava, Quaternionic structures on a manifold and subordinated structures, Annali di Mat. Pura e Appl. (4) 171 (1996), 205-273.

[4] D.V.Alekseevsky, S.Marchiafava, M.Pontecorvo, Compatible complex structures on almost quaternionic manifolds, Trans. Amer. Math. Soc. 351 (1999), 997-1014.

[5] B. Alexandrov, G. Grantcharov, S. Ivanov, Curvature properties of twistor spaces of quaternionic Kähler manifolds, Journal of Geometry 62 (1998), 1-12.

[6] B.Alexandrov, S.Ivanov, Vanishing theorems on Hermitian manifolds, Diff. Geom. Appl., 14 (3) (2001), 251-265.

[7] M. F. Atiayah, N. Hitchin, I.M. Singer, Self-duality in four-dimensional Riemannian geometry, Proc. Roy. Soc. London Ser. A, 362(1978), 425 - 461.

[8] M.Berger, Remarques sur le grupe d'holonomie des variétés Riemanniennes, C.R. Acad. Sci. Paris 262 (1966), 1316-1318.

[9] A.Besse, Einstein manifolds, Springer-Verlag, New York, 1987.

[10] J.-M. Bismut, A local index theorem for non-Kähler manifolds, Math. Ann. 284 (1989), 681 699.

[11] E.Bonan, Sur les G-structures de type quaternionen, Cahiers de Topologie et Géométrie Differentialle, 9 (1967), 389-461. 
[12] R. Bryant, Lie groups and twistor spaces, Duke Math. J., Vol. 52,1(1985), 223 - 261.

[13] J. Davidov, O. Muškarov, Twistor spaces with Hermitian Ricci tensor, Proc. Amer. Math. Soc. 109(1990), $1115-1120$.

[14] I. Dotti, A. Fino, Hypekähler torsion structure invariant by nilpotent Lie groups, math.DG/0112166.

[15] J. Eells, S. Salamon, Constructions twistorielles des applications harmoniques, C. R. Acad. Sc. Paris, 296(1983), $685-687$.

[16] Th. Friedrich, S.Ivanov, Parallel spinors and connections with skew symmetric torsion in string theory, math.DG/0102142.

[17] Th. Friedrich, S.Ivanov, Almost contact manifolds and type II string equations, math.DG/0111131.

[18] S.J.Gates, C.M.Hull, M.Roěk, Twisted multiplets and new supersymmetric non-linear $\sigma$ models, Nucl. Phys. B248(1984), 157 - 186.

[19] P.Gauduchon, La 1-forme de torsion d'une variété hermitienne compacte, Math. Ann. 267 (1984), $495-518$.

[20] P. Gauduchon, Hermitian connections and Dirac operators, Bol. U. M. I. ser. VII, vol. XI-B, supl. 2 (1997), $257-289$.

[21] G.Grantcharov, Y.S.Poon, Geometry of Hyper-Kähler connections with torsion, Comm. Math. Phys. 213 (2000), 19-37.

[22] A. Gray, L. Hervela, The sixteen classes of almost Hermitian manifolds and their linear invariants, Ann. Math. Pura e App., 123,35 - 58(1980).

[23] N.J.Hitchin, A.Karlhede, U.Lindström, M.Roček, Hyperkähler metrics and supersymmetry, Commun. Math. Phys. 108 (1987), 535-589.

[24] P.S.Howe, G.Papadopoulos, Further remarks on the geometry of two dimensional nonlinear $\sigma$ models, Class. Quantum Grav. 5 (1988), 1647-1661.

[25] P.S.Howe, G.Papadopoulos, Twistor spaces for hyper-Kähler manifolds with torsion, Phys. Lett. B379(1996), $81-86$.

[26] P.S.Howe, A.Opfermann, G.Papadopoulos, Twistor spaces for QKT manifolds, Comm. Math. Phys. 197(1998), $713-727$..

[27] S.Ishihara, Quaternion Kählerian manifolds, J. Diff. Geom. 9, (1974), 483-500.

[28] S. Ivanov, Geometry of Quaternionic Kähler connections with torsion, math.DG/0003214, to appear in J. Geom. Phys.

[29] S. Ivanov, Connections with torsion, parallel spinors and geometry of Spin(7) manifolds, math.DG/0111216

[30] S.Ivanov, G. Papadopoulos, Vanishing theorems and string backgrounda, Class. Quant. Grav. 18 (2001), 1089-1110. 
[31] S. Kobayashi, K. Nomizu, Foundations of Differential Geometry, 2 volumes, Interscience Publ., New York, 1963, 1969.

[32] J. Michelson, A. Strominger, The geometry of superconformal quantum mechanics, Comm. Math. Phys. 213 (2000), 1-17.

[33] H.Nishino, Alternative $N=(4,0)$ superstring and $\sigma$-models, Phys. Lett. B 355 (1995), 117126.

[34] M.Obata, Affine connections on manifolds with almost complex quaternionic or Hermitian structures, Jap. J. Math. 26 (1956), 43-79.

[35] A.Opfermann, G.Papadopoulos, Homogeneous HKT and QKT manifolds, e-print, http://xxx.lanl.gov, math-ph/9807026.

[36] G.Papadopoulos, (2,0) supersymmetric sigma models and almost complex structures, Nucl.Phys. B448 (1995), 199-219

[37] H. Pedersen, Y.-S. Poon, A. Swann, Hypercomplex structure associated to quaternionic manifolds, Diff. Geom. Appl. 9 (1998), 273-292.

[38] Y.-S. Poon, A. Swann Potential functions on HKT spaces, Clas. Quantum Grav. 18 (2001), 4711-4714.

[39] Y.-S. Poon, A. Swann, Superconformal symmetry and Hypekähler manifolds with torsion, math.DG/0111276.

[40] S.Salamon, Quaternionic Kähler manifolds, Invent. Math. 67 (1982), 143-171.

[41] S.Salamon Special structures on four manifold, Riv. Math. Univ. Parma (4) 17 (1991), 109-123.

[42] S.Salamon, Differential geometry on quaternionic Kähler manifolds, Ann. Sci. Ec. Norm. Sup. Ser (4) 19 (1986), 31-55.

[43] S.Salamon, Riemannian geometry and holonomy groups, Pitman Research Notes in Math. Series, vol. 201 (Longman, New-York, 1989).

[44] K. Sekigawa, Almost Hermitian structures on twistor bundles, Proc. Ramanujan Centennial Int. Conf., Annamalainagar (1987), 127 - 136.

[45] A. Strominger, Superstrings with torsion, Nucl. Physics B 274 (1986), 254-284.

[46] A. Swann, HyperKähler and quaternionic Kähler geometry, Math. Ann. 289 (1991), 421-450.

Authors' address: Stefan Ivanov, Ivan Minchev University of Sofia, Faculty of Mathematics and Informatics, Department of Geometry, 5 James Bourchier blvd, 1164 Sofia, BULGARIA.

E-mail: ivanovsp@fmi.uni-sofia.bg 